\title{
Stochastic Modeling of Coherent Wave Fields over Variable Depth
}

\author{
P. B. SMIT* \\ Delft University of Technology, Delft, Netherlands \\ T. T. JANSSEN* AND T. H. C. HERBERS* \\ Theiss Research, El Granada, California
}

(Manuscript received 23 October 2014, in final form 22 January 2015)

\begin{abstract}
Refractive focusing of swell waves can result in fast-scale variations in the wave statistics because of wave interference, which cannot be resolved by stochastic wave models based on the radiative transport equation. Quasi-coherent statistical theory does account for such statistical interferences and the associated wave inhomogeneities, but the theory has thus far been presented in a form that appears incompatible with models based on the radiative transfer equation (RTE). Moreover, the quasi-coherent theory has never been tested against field data, and it is not clear how the coherent information inherent to such models can be used for better understanding coastal wave and circulation dynamics. This study therefore revisits the derivation of quasi-coherent theory to formulate it into a radiative transport equation with a forcing term that accounts for the inhomogeneous part of the wave field. This paper shows how the model can be nested within (or otherwise used in conjunction with) quasi-homogeneous wave models based on the RTE. Through comparison to laboratory data, numerical simulations of a deterministic model, and field observations of waves propagating over a nearshore canyon head, the predictive capability of the model is validated. The authors discuss the interference patterns predicted by the model through evaluation of a complex cross-correlation function and highlight the differences with quasi-homogeneous predictions. These results show that quasi-coherent theory can extend models based on the RTE to resolve coherent interference patterns and standing wave features in coastal areas, which are believed to be important in nearshore circulation and sediment transport.
\end{abstract}

\section{Introduction}

The evolution of wind-driven surface waves on the open ocean is largely determined by the action of wind (Phillips 1957; Miles 1957), dissipation (white capping; Hasselmann 1974), and third-order nonlinear effects (or quadruplet wave-wave interactions; Hasselmann 1962). Over the continental shelves and near the coast, ocean waves are affected by many additional processes, including refraction by depth and current variations (e.g., Munk and Traylor 1947; O'Reilly and Guza 1993; Dodet et al. 2013; Pearman et al. 2014); Bragg scattering by

\footnotetext{
* Current affiliation: NorthWest Research Associates, El Granada California.
}

Corresponding author address: P. B. Smit, NorthWest Research Associates, P.O. Box 1533, El Granada, CA 94018.

E-mail: pieterbartsmit@gmail.com bottom irregularities (e.g., Long 1973; Ardhuin and Herbers 2002); bottom friction (e.g., Grant and Madsen 1979; Ardhuin et al. 2003); and eventually, when approaching the shoreline, second-order nonlinearity (e.g., Freilich and Guza 1984; Kaihatu and Kirby 1995; Eldeberky 1996; Herbers and Burton 1997; Agnon and Sheremet 1997; Janssen et al. 2006), depth-induced breaking (e.g., Battjes and Janssen 1978; Thornton and Guza 1983; Apotsos et al. 2008; Salmon et al. 2015), and wave reflection from shore (e.g., Elgar et al. 1994). Although some of these processes can be highly nonlinear and are not all well understood (e.g., depth-induced wave breaking and white capping), they generally drive slow variations in the mean wave statistics such that the evolution of the wave variance density spectrum $E(\mathbf{k}, \mathbf{x}, t)$ through time $t$, geographical space $\mathbf{x}=\left(x_{1}, x_{2}\right)$, and wavenumber space $\mathbf{k}=\left(k_{1}, k_{2}\right)$ can be described by the radiative transport equation (RTE):

$$
\partial_{t} E+\mathbf{c}_{x} \cdot \nabla_{\mathbf{x}} E+\mathbf{c}_{k} \cdot \nabla_{\mathbf{k}} E=S .
$$


Here, $\partial_{t}$ is shorthand for partial differentiation with respect to the subscript variables $\nabla_{\mathbf{x}} \equiv\left(\partial_{x_{1}}, \partial_{x_{2}}\right)$ and $\nabla_{\mathbf{k}} \equiv\left(\partial_{k_{1}}, \partial_{k_{2}}\right)$. The left side of Eq. (1) represents the conservation of wave energy in a slowly varying medium, with $\mathbf{c}_{x}=\left(c_{x_{1}}, c_{x_{2}}\right)$ and $\mathbf{c}_{k}=\left(c_{k_{1}}, c_{k_{2}}\right)$ denoting transport velocities through geographic and spectral space, respectively (Willebrand 1975), and the forcing term $S(\mathbf{k}, \mathbf{x}, t)$ on the right of Eq. (1) represents source term contributions to account for nonconservative and cross-spectral transfers. The continuing development of models based on the RTE has focused mostly on improvement in the source terms, which for the most part are based on parameterized physical models that require empirical tuning and can contribute large uncertainties to model predictions.

Apart from the usual WKB assumptions for a slowly varying medium, the use of the RTE requires that wave components are statistically independent (Komen et al. 1994), which is valid for quasi-homogeneous (and Gaussian) wave fields. In deep water, where the evolution is mostly dominated by the source term balance, this is generally true. However, in shallow water, where the waves interact with slowly varying topography (or currents), the coherent scattering of narrowband wave fields can result in inhomogeneities and fast-scale variations in wave energy (Janssen et al. 2008), which can have an $O(1)$ effect on the mean statistics (Smit and Janssen 2013, hereinafter SJ13) and-as a consequence-affect wavedriven circulation and transport processes. These effects are not accounted for in models based on the RTE.

The quasi-coherent (QC) theory presented in SJ13 is a fundamental generalization of the RTE to account for coherence (which for constant depth was also considered by Pedersen and Lokberg 1992) and incorporates inhomogeneities and scattering effects by resolving (and transporting) cross-phase information in the wave field statistics. However, the form of the model equations as derived in SJ13 [see Eqs. (15) and (16) in SJ13] is quite different from the conventional RTE-type transport models, which hamper the physical interpretation of the inhomogeneity contributions and make it more difficult to combine the two different modeling approaches in a single numerical model. As part of this work we revisit and expand on the theoretical results from SJ13 to show that the QC approximation can be written in a similar form as Eq. (1), but with an additional scattering term $S_{\mathrm{QC}}$ on the RHS, that describes spatial energy variations resulting from wave interference and depends on local cross correlations between spectral wave components.

We further develop a consistent numerical implementation for this model, validate the model against laboratory and field observations, and explore the interpretation and use of the cross correlators implicit in the coupled-mode (CM) spectrum (as opposed to the variance density spectrum). In that regard, we briefly summarize the principal results from SJ13 (section 2) and derive and discuss a consistent approximation for medium variations based on the wave field decorrelation length scale (section 2). We present simulations with the new model of laboratory flume experiments (section 3) and field observations of ocean waves interacting with a submarine canyon (section 4), discuss the effects of wave inhomogeneity in the observational data and model results, and sum up our principal findings in section 5.

\section{Evolution of inhomogeneous wave fields}

To describe the evolution of inhomogeneous surface wave statistics in a variable medium, we consider the transport of what we refer to as the CM spectrum, ${ }^{1}$ defined as

$$
\mathscr{E}(\mathbf{k}, \mathbf{x}, t)=\mathscr{F}_{\xi, \mathbf{k}}[\Gamma(\mathbf{x}+\boldsymbol{\xi} / 2, \mathbf{x}-\boldsymbol{\xi} / 2, t)],
$$

where $\mathscr{F}_{\xi, \mathbf{k}}[\ldots]$ denotes the Fourier transform from spatial lag $\boldsymbol{\xi}$ to wavenumber $\mathbf{k}$ (see appendix A), and the correlation function $\Gamma$ of a wave field variable $\zeta$ between two spatial points $\mathbf{x}$ and $\mathbf{x}^{\prime}$ is defined as

$$
\Gamma\left(\mathbf{x}, \mathbf{x}^{\prime}, t\right)=\frac{1}{2}\left\langle\zeta(\mathbf{x}, t) \zeta^{*}\left(\mathbf{x}^{\prime}, t\right)\right\rangle .
$$

Here, $\zeta(\mathbf{x}, t)$ is a complex, zero-mean, Gaussian variable, of which the real part is the surface elevation $\eta(\mathbf{x}, t)=\operatorname{Re}[\zeta(\mathbf{x}, t)]$ and the imaginary part is its Hilbert transform (e.g., Mandel and Wolf 1995); $\langle\ldots\rangle$ denotes the ensemble average; and $\zeta^{*}$ represents the complex conjugate of $\zeta$. The CM spectrum represents the complete second-order wave statistics, including crossvariance contributions. It is related to the bulk wave variance $\mathscr{V}(\mathbf{x}, t)=\Gamma(\mathbf{x}, \mathbf{x}, t)$ through its marginal distribution $\mathscr{V}(\mathbf{x}, t)=\int \mathscr{E}(\mathbf{k}, \mathbf{x}, t) d \mathbf{k}$, which consequently is strictly positive. However, the CM spectrum is not pointwise positive (it can have negative contributions) and should thus not be confused with a variance density spectrum (see SJ13).

In the absence of sources or sinks of wave energy, and under the assumption that the wave field consists of progressive plane surface gravity waves propagating through a slowly varying medium, such that the wavenumber $k$ and angular frequency $\omega$ are related by a linear dispersion relation, an evolution equation for the coupled-mode spectrum $\mathscr{E}$ can be derived (see SJ13):

\footnotetext{
${ }^{1}$ Also known as the Wigner or Wigner-Ville spectrum (see, e.g., Wigner 1932; Ville 1948).
} 


$$
\begin{aligned}
& \partial_{t} \mathscr{E}(\mathbf{k}, \mathbf{x}, t) \\
& \quad=-\mathrm{i} \int \hat{\Omega}\left(\mathbf{k}, \mathbf{q},-\mathrm{i} \nabla_{\mathbf{x}}\right) \mathscr{E}\left(\mathbf{k}-\frac{\mathbf{q}}{2}, \mathbf{x}\right) \exp [\mathrm{iq} \cdot \mathbf{x}] d \mathbf{q}+*
\end{aligned}
$$

where $\hat{\Omega}$ denotes the integration kernel that operates on $\mathscr{E}$, the wavenumber $\mathbf{q}=\left(q_{1}, q_{2}\right)$ is associated with spatial variations of the medium, and $*$ denotes the complex conjugate.

In the linear approximation, the dispersion relation $\sigma(k, h)$ for ocean surface gravity waves that relates $\omega=\sigma(k, h)$ and $k(\mathbf{k})=|\mathbf{k}|$ is

$$
\sigma(k, h)=\sqrt{g k \tanh (k h)},
$$

with $g\left(=9.81 \mathrm{~m}^{2} \mathrm{~s}^{-1}\right)$ as the gravitational acceleration, and $h(\mathbf{x})$ as the mean depth. In this case the integration kernel $\hat{\Omega}$ in Eq. (6) is defined as

$$
\hat{\Omega}\left(\mathbf{k}, \mathbf{q},-\mathrm{i} \nabla_{\mathbf{x}}\right)=\hat{\sigma}-\frac{\mathrm{i}}{2} \hat{\sigma}_{k} \tilde{\mathbf{k}} \cdot \nabla_{\mathbf{x}}
$$

Here, $\hat{\sigma}(\mathbf{k}, \mathbf{q})$ denotes the spatial Fourier transform $\left(\mathbf{x}^{\prime} \rightarrow \mathbf{q}\right)$ of the dispersion relation $\sigma(\mathbf{k}, \mathbf{x})=\sigma[k, h(\mathbf{x})]$, such that $\hat{\sigma}(\mathbf{k}, \mathbf{q})=\mathscr{F}_{\mathbf{x}^{\prime}, \mathbf{q}}\left[\sigma\left(\mathbf{k}, \mathbf{x}^{\prime}\right)\right]$. Furthermore, in Eq. (6), $\hat{\sigma}_{k}$ is short for $\partial_{k} \hat{\sigma}$, and $\tilde{\mathbf{k}}=\mathbf{k} / k$.

\section{Medium variations in a coherent wave field}

Combined, Eqs. (4) and (6) summarize the principal theoretical result from SJ13 and represent the starting point of this work. In the following, we will derive a consistent form of this model using the decorrelation length scale of the wave field, making the physical interpretation more intuitive, relating it explicitly to the RTE, and making it suitable for numerical evaluation. In particular, the Fourier transform on the right side of
Eq. (4) makes the evolution of the coupled-mode spectrum dependent on medium variations throughout the entire spatial domain. Since random ocean waves have a finite decorrelation length scale, this is not only impractical but also unnecessary from a physical point of view.

To make this explicit, we consider that the slow medium variations are characterized by a small parameter $\varepsilon \ll 1$, so that the medium varies $O(1)$ over distances $L_{0} / \varepsilon$, with $L_{0}$ being a characteristic wavelength. Inhomogeneities in the wave field induced through medium variations cause $O(1)$ variations in the wave field statistics on the scale $L_{0} / \mu$, with $\mu \ll 1$ being a measure of the wavenumber mismatch between coherent components. The width of the spectrum is measured by the parameter $\delta=\Delta k / k_{0}$, where $\Delta k$ is a characteristic width and $k_{0}=2 \pi / L_{0}$. The latter is used to define a coherent length scale as $\xi_{c}=L_{0} / \delta$, so that $\Gamma(\boldsymbol{\xi}, \mathbf{x}) \rightarrow 0$ as $|\boldsymbol{\xi}| \rightarrow \xi_{c}$. For narrowband waves $\delta \ll 1$, implying that the wave field remains correlated over many wavelengths. To relate the coherent radius to the variations in the medium, we consider the ratio $\beta=\varepsilon / \delta$, such that for $\beta \ll 1$ changes in topography occur over distances much larger than the decorrelation scale, whereas for $\beta=O(1)$ significant changes occur within the coherent radius of the wave field. In the latter case, the wave field retains the memory of the wave-bottom interaction and coherent effects can be important. Note that $\beta$ can become $O(1)$ even if the medium is slowly varying relative to the length scale of the individual waves (thus $\varepsilon \ll 1$ ).

To illustrate how the coherent radius constrains the effect of medium variations, and thus derive a consistent and local approximation, we write Eq. (4) as

$$
\partial_{t} \mathscr{E}(\mathbf{k}, \mathbf{x}, t)=G(\mathbf{k}, \mathbf{x}, t)+*
$$

where

$$
G=\frac{-\mathrm{i}}{(4 \pi)^{4}} \int \Omega\left(\mathbf{k}, \mathbf{x}+\mathbf{x}^{\prime},-\mathrm{i} \nabla_{\mathbf{x}}\right) \Gamma(\boldsymbol{\xi}, \mathbf{x}) \exp \left[\mathrm{iq} \cdot\left(\frac{1}{2} \boldsymbol{\xi}-\mathbf{x}^{\prime}\right)\right] \exp [-\mathrm{ik} \cdot \boldsymbol{\xi}] d \boldsymbol{\xi} d \mathbf{x}^{\prime} d \mathbf{q}
$$

Here, we replace $\hat{\Omega}\left(\mathbf{k}, \mathbf{q},-\mathrm{i} \nabla_{\mathbf{x}}\right)$ and $\mathscr{E}(\mathbf{k}, \mathbf{x})$ by $\mathscr{F}_{\mathbf{x}^{\prime \prime}, \mathbf{q}}\left[\Omega\left(\mathbf{k}, \mathbf{x}^{\prime \prime},-\mathrm{i} \nabla_{\mathbf{x}}\right)\right]$ and $\mathscr{F}_{\xi, \mathbf{k}}[\Gamma(\boldsymbol{\xi}, \mathbf{x})]$, respectively, and we changed the variables to $\mathbf{x}^{\prime}=\mathbf{x}^{\prime \prime}-\mathbf{x}$.

For waves with a finite coherent radius $\xi_{c}$, the field decorrelates $(\Gamma \rightarrow 0)$ when $|\boldsymbol{\xi}|>\xi_{c}$ so that contributions to the integral in Eq. (8) are limited to the extent of the coherent radius. Further, oscillatory behavior of the integral over $\mathbf{q}$ implies that contributions to the integral only emerge (in the form of Dirac delta functions) along curves for which $(1 / 2) \xi-\mathbf{x}^{\prime}=0$. In other words, the evolution of the statistics is only affected by the topography within a radius $\xi_{c} / 2$ around $\mathbf{x}$, or the region of statistical dependence, as would be expected on physical grounds.

Although here we make such approximations explicitly, the same approximation is implicit in the RTE. If we assume that the wave field is relatively broad banded with a small coherent radius (relative to the medium variations), we have that $O(\beta)=O(\varepsilon) \ll 1$. In this case, the wave field decorrelates before significant changes in 
the topography occur, and it is reasonable to express the medium variability as a Taylor expansion in $\mathbf{x}^{\prime}$ around $\mathbf{x}^{\prime}=0$. This implies that $\sigma$ and $\sigma_{k}$ in $\Omega$ are replaced by their respective series expansions, and retaining terms up to $O(\beta)$, we obtain

$$
\begin{aligned}
\Omega^{\mathrm{RTE}}\left(\mathbf{k}, \mathbf{x}, \mathbf{x}^{\prime},-\mathrm{i} \nabla_{\mathbf{x}}\right)= & \sigma-\mathbf{x}^{\prime} \cdot \mathbf{c}_{k} \\
& -\frac{\mathrm{i}}{2}\left[\mathbf{c}_{x}+\left(\mathbf{x}^{\prime} \cdot \sigma_{k, h} \nabla_{\mathbf{x}} h\right) \tilde{\mathbf{k}}\right] \cdot \nabla_{\mathbf{x}} .
\end{aligned}
$$

Here, $\mathbf{c}_{k}(\mathbf{k}, \mathbf{x})=-\sigma_{h} \nabla_{\mathbf{x}} h$ and $\mathbf{c}_{x}=\tilde{\mathbf{k}} \sigma_{k}$ with $\sigma_{h}(\mathbf{k}, \mathbf{x})=$ $\left.\partial_{h} \sigma\right|_{k(\mathbf{k}), h(\mathbf{x})}$ and $\sigma_{k h}(\mathbf{k}, \mathbf{x})=\left.\partial_{h} \sigma_{k}\right|_{k(\mathbf{k}), h(\mathbf{x})}$. Substituting the local approximation Eq. (9) into Eq. (8), we can show that Eq. (7) reduces to the RTE, as in

$$
\partial_{t} \mathscr{E}=G^{\mathrm{RTE}}+*=-\mathbf{c}_{k} \cdot \nabla_{\mathbf{k}} \mathscr{E}-\mathbf{c}_{x} \cdot \nabla_{\mathbf{x}} \mathscr{E} .
$$

Note that in this approximation, the transport velocities are determined by the mean depth and local bottom slope, which is equivalent to assuming that the local bathymetry is effectively planar. By making this approximation explicit here, we show that the RTE is merely a special case of the transport Eq. (7), emerging when we assume that the wave field decorrelates on a much faster scale than the medium varies $(\beta \ll 1)$, in which case only the local bathymetric features are relevant to the evolution of the wave field statistics. In reality, wave fields described by the RTE have a finite spectral width and coherent radius and whether the assumptions implied by the RTE are reasonable depends entirely on the nature of the medium variations and the width of the spectrum.

To write the general transport Eq. (7) in a form similar to the RTE, but with an additional source term that accounts for the development of inhomogeneities by medium variations (e.g., coherent interferences), we approximate $\Omega$ as a superposition of the RTE operator and a remainder:

$$
\begin{aligned}
\Omega\left(\mathbf{k}, \mathbf{x}+\mathbf{x}^{\prime},-\mathrm{i} \nabla_{\mathbf{x}}\right) \approx & \Omega^{\mathrm{RTE}}\left(\mathbf{k}, \mathbf{x}, \mathbf{x}^{\prime},-\mathrm{i} \nabla_{\mathbf{x}}\right) \\
& +\Delta \Omega\left(\mathbf{k}, \mathbf{x}, \mathbf{x}^{\prime},-\mathrm{i} \nabla_{\mathbf{x}}\right) .
\end{aligned}
$$

Assuming that $\beta=O(1)$ so that the field remains coherent on the scale that the medium undergoes $O(1)$ changes, but the evolution of the local statistics remains unaffected by the far-field representation of $\Omega$ (for $\left|\mathbf{x}^{\prime}\right| \geq \xi_{c}$ ), we define $\Delta \Omega$ within the coherent region as

$$
\Delta \Omega\left(\mathbf{k}, \mathbf{x}, \mathbf{x}^{\prime},-\mathrm{i} \nabla_{\mathbf{x}}\right)=W\left[\Omega-\Omega^{\mathrm{RTE}}\right] \quad \text { for } \quad\left|x_{j}^{\prime}\right| \leq \xi_{c} / 2,
$$

where $W\left(\mathbf{x}^{\prime}\right)$ is a window function (see appendix B) that smoothly transitions from unity to zero as $\left|x_{j}^{\prime}\right| \rightarrow \xi_{c}$ so that near the extremities of the domain (where the wave field is nearly decorrelated) the far-field representation of $\Omega$ is relaxed to $\Omega^{\mathrm{RTE}}$. Also, to be consistent with use of the Fourier integrals in Eq. (7), $\Delta \Omega$ is presumed spatially periodic outside of the coherent domain. With this, we can write Eq. (7) as $\partial_{t} \mathscr{E}=G_{\mathrm{RTE}}+S_{\mathrm{QC}}+*$, and thus

$$
\partial_{t} \mathscr{E}+\mathbf{c}_{k} \cdot \nabla_{\mathbf{k}} \mathscr{E}+\mathbf{c}_{x} \cdot \nabla_{\mathbf{x}} \mathscr{E}=S_{\mathrm{QC}}+* .
$$

Here, the scattering term $S_{\mathrm{QC}}$ is expressed in terms of the $\mathrm{CM}$ spectrum as

$$
S_{\mathrm{QC}}=-\mathrm{i} \int \Delta \hat{\Omega}\left(\mathbf{k}, \mathbf{x}, \mathbf{q},-\mathrm{i} \nabla_{\mathbf{x}}\right) \mathscr{E}\left(\mathbf{k}-\frac{1}{2} \mathbf{q}, \mathbf{x}\right) d \mathbf{q},
$$

with $\Delta \hat{\Omega}=\mathscr{F}_{\mathbf{x}^{\prime}, \mathbf{q}}(\Delta \Omega)$. In discrete form, where we use the discrete transform $\Delta \hat{\Omega}^{\mathbf{q}^{\prime}}=\overline{\mathscr{F}}_{\mathbf{x}^{\prime}, \mathbf{q}^{\prime}}(\Delta \Omega)$ so that $\Delta \hat{\Omega}=\sum_{\mathbf{q}^{\prime}} \delta\left(\mathbf{q}-\mathbf{q}^{\prime}\right) \Delta \hat{\Omega}^{\mathbf{q}^{\prime}} \quad$ with $\quad \mathbf{q}^{\prime}=\left[m_{1}^{q} \Delta q, m_{2}^{q} \Delta q\right]$ and $\Delta q=2 \pi / \xi_{c}$, we thus have

$$
\begin{aligned}
\partial_{t} \mathscr{E} & +\mathbf{c}_{k} \cdot \nabla_{\mathbf{k}} \mathscr{E}+\mathbf{c}_{x} \cdot \nabla_{\mathbf{x}} \mathscr{E} \\
& =-\mathrm{i} \sum_{\mathbf{q}} \Delta \hat{\Omega}^{\mathbf{q}}\left(\mathbf{k}, \mathbf{x},-\mathrm{i} \nabla_{\mathbf{x}}\right) \mathscr{E}(\mathbf{k}-\mathbf{q} / 2, \mathbf{x})+*
\end{aligned}
$$

where $\mathbf{q}=\mathbf{q}^{\prime}$. Equation (15) is a consistent representation of the quasi-coherent theory developed by SJ13 for variable bathymetry. It introduces no new assumptions but uses the decorrelation length scale inherent to the wave field to rewrite the equations into an RTE-like form, making physical interpretations of the theory more intuitive and allowing it to be readily numerically evaluated using similar numerical schemes as commonly used for RTE-type models.

In what follows, we will refer to Eq. (15) as the quasicoherent model (QCM). In the simulations presented in this work, we consider steady-state solutions to Eq. (15) (such that $\partial_{t} \mathscr{E}=0$ ) computed on a discrete grid by means of a finite-difference approximation based on the solution technique for the RTE as found in Booij et al. (1999). For details on the numerical implementation, we refer to appendix B.

\section{Wave deformation by an elliptical shoal}

A monochromatic, initially unidirectional wave field that interacts with topography can be considered as the archetype of a coherent scattering problem. Moreover, it represents an excellent test on the limits of the stochastic model since the QCM explicitly assumes a finite coherent radius (or finite $\Delta q$ ) and thus a finite-width spectrum.

To illustrate the behavior of the QCM and the statistical information that is inherently available in the model, and test its performance under such conditions, we consider the wave basin experiment by Berkhoff et al. (1982), where monochromatic (period 1s), unidirectional waves (wave height $H=0.0464 \mathrm{~m}$ ) were generated at the wavemaker (at $x=-10$, depth $0.45 \mathrm{~m}$ ) and 
propagated over a shoal (crest located at $\mathbf{x}=0 \mathrm{~m}$, depth of $0.135 \mathrm{~m}$ ) situated on a 1:50 slope (see Fig. 1). Wave heights were measured along eight transects at regular intervals, of which we consider three (indicated in Fig. 1).

The RTE and QCM are numerically evaluated on a rectangular spatial $\left(20 \times 20 \mathrm{~m}^{2}\right)$ and spectral domain $\left[10 \times 10 \mathrm{rad}^{2} \mathrm{~m}^{-2}\right.$, starting at $\left.\mathbf{k}=(-0.05,-5) \mathrm{rad} \mathrm{m}^{-1}\right]$, uniformly discretized with mesh sizes $\Delta x=\Delta y=5 \mathrm{~cm}$ and $\Delta k_{x}=\Delta k_{y}=0.1 \mathrm{rad} \mathrm{m}^{-1}$. The finite bandwidth $\Delta q=$ $0.2 \mathrm{rad} \mathrm{m}^{-1}$ implies that the maximum resolvable coherent scale in the model is $\xi_{c}=31.4 \mathrm{~m}$, and we include components $\mathbf{q}$ with wavenumbers smaller than $k_{p} / 2$, where $k_{p}$ is the peak wavenumber of the incident wave field. To simulate a monochromatic wave field, the model is initiated at the boundary with a Gaussian-shaped wave spectrum $\mathscr{E}$, with the spectral peak at $k_{p}=4.21 \mathrm{rad} \mathrm{m}^{-1}$ and a standard deviation of $\Delta k_{\text {spec }}=0.2 \mathrm{rad} \mathrm{m}^{-1}$, which effectively corresponds to a narrowband long-crested wave field with a width of $0.1 \mathrm{~Hz}$ and $1.5^{\circ}$ in frequency and directional space, respectively.

To augment the observations with data where no observations are available, we include model simulations with the deterministic model Surface Waves till Shore (SWASH; Zijlema et al. 2011), which solves the 3D Euler equations for a free-surface fluid of constant density. This highly detailed model reproduces the laboratory observations in great detail (see, e.g., Stelling and Zijlema 2003), and we use it here to provide a ground truth for the QCM to validate its ability to capture wave interferences and its representation of the complete second-order statistics. Since the CQ model is linear, the SWASH model is linearized also by reducing the incident wave height to $H=0.001 \mathrm{~m}$. In this way we

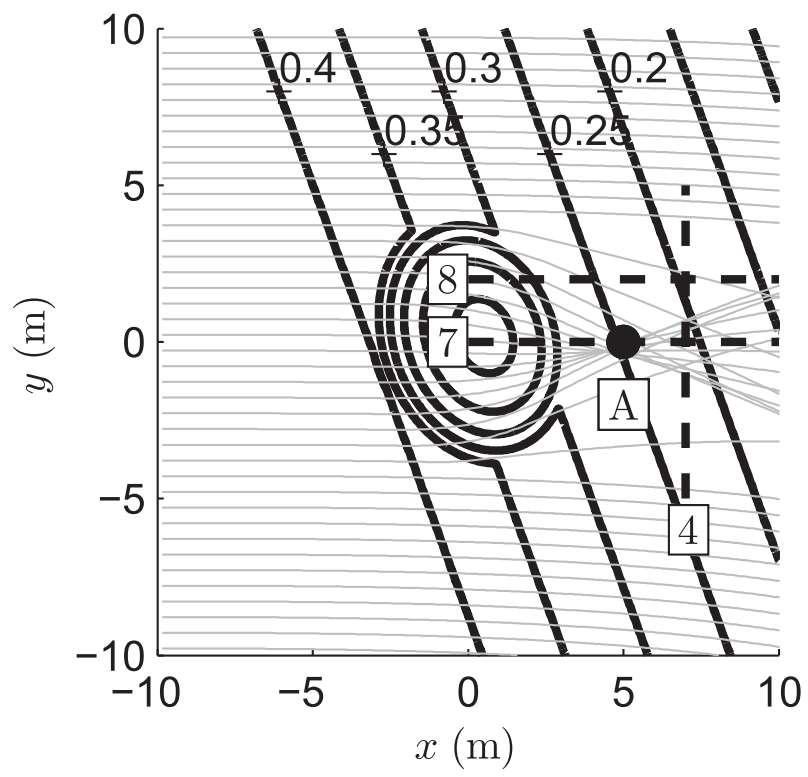

FIG. 1. Plan view of the experimental setup by Berkhoff et al. (1982), including ray trajectories for unidirectional monochromatic waves (thin gray lines) that start at $x=-10 \mathrm{~m}$ and propagate toward the shoal. Depth contours are indicated by the black solid lines; instrumented transects used in this study (identified by boxed numerals) are indicated by dashed lines, whereas the solid dot (identified by the boxed A) indicates the point A discussed in the main text.

have a direct comparison with the QCM and can identify nonlinear effects in the observations.

\section{Results}

The refractive focusing of the waves produces a lateral interference pattern in the wake of the shoal (e.g., Fig. 1). The finescale pattern is reproduced by the QCM, and normalized wave heights correspond well
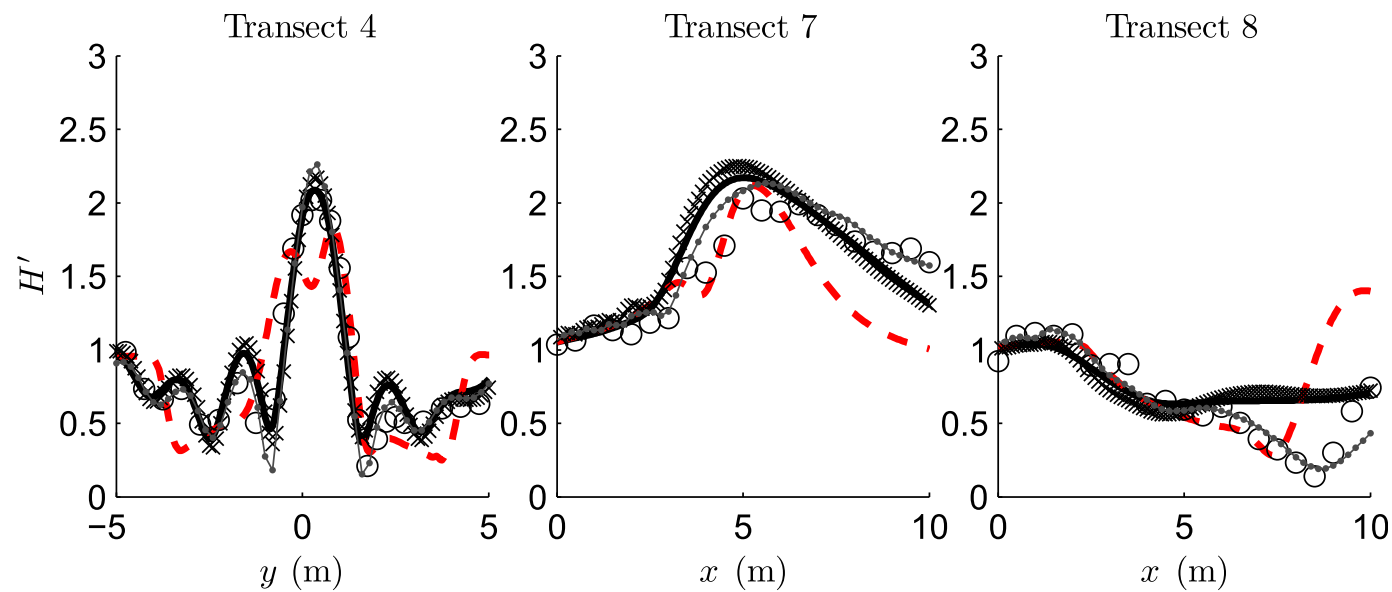

FIG. 2. The normalized wave height $H^{\prime}$ (normalized with incident wave height) along the indicated transects. Comparison between observations (open circles), and relative wave heights obtained with the QCM (solid line), RTE (dashed line), and (linearized) SWASH (crosses). For reference, results from the fully nonlinear SWASH model forced with the actual wave height are also included (gray dotted lines). 

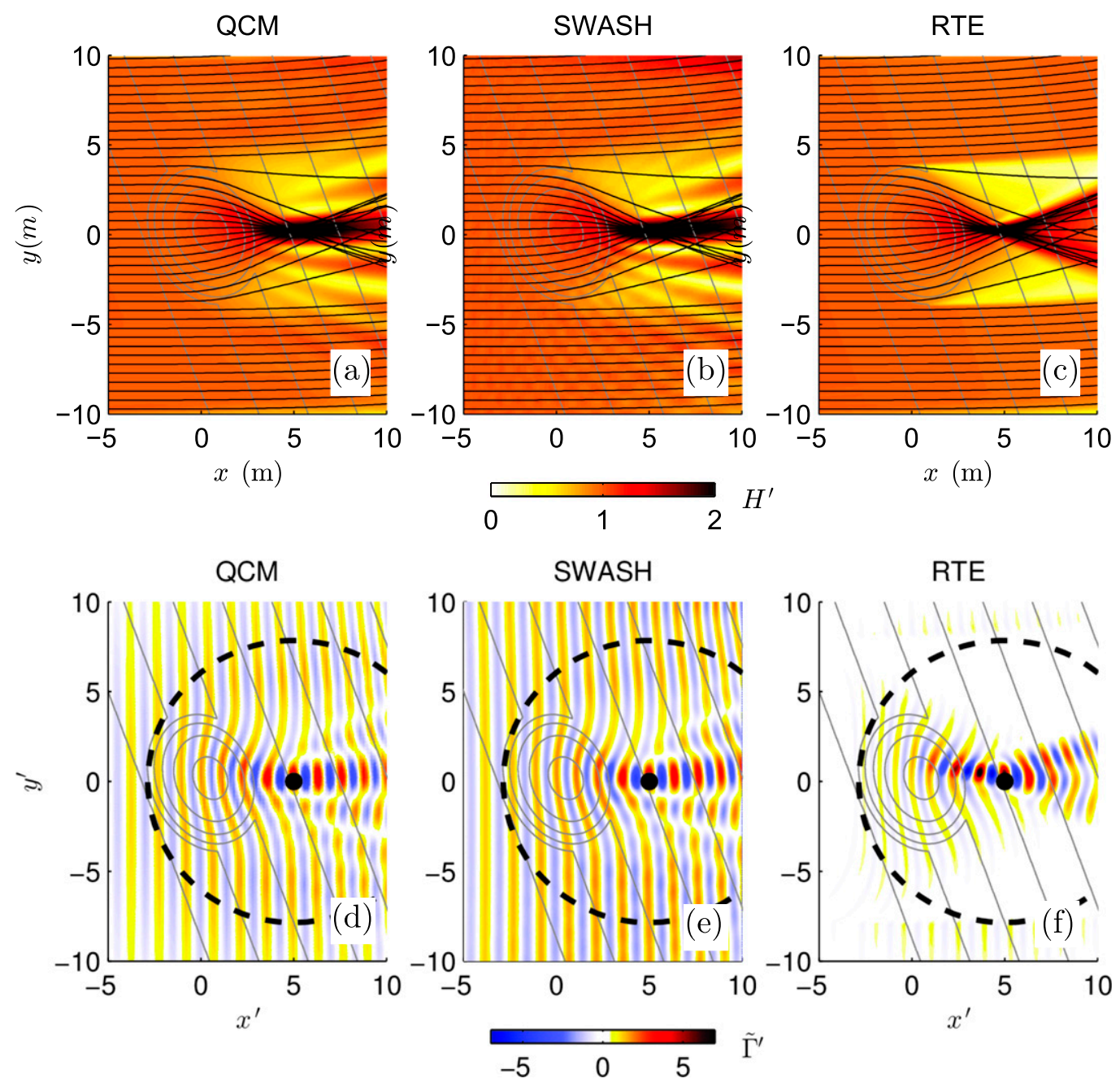

FIG. 3. Plan view of (a)-(c) normalized wave heights $H^{\prime}$ and (d)-(f) normalized covariance function $\tilde{\Gamma}_{\mathbf{x}_{A}}^{\prime}\left(\mathbf{x}^{\prime}\right)$ obtained using the QCM, RTE, and the "linear" SWASH model for the experimental setup by Berkhoff et al. (1982). Gray lines indicate bottom contours, while the black lines are ray trajectories. The wave height and covariance functions are normalized with the wave height and variance at the boundary, respectively. The dot in the lower panels corresponds to point $\mathbf{x}_{A}$, and the circle has a radius of $\xi_{c} / 4$.

with observations (Fig. 2). In contrast, the quasihomogeneous model (RTE) underestimates wave heights along central transect 7 and does not reproduce the wave heights in transect 4 . These shortcomings have been noted earlier by O'Reilly and Guza (1991) in comparisons of a ray-based spectral refraction model (equivalent to RTE) and a phase-resolved refraction-diffraction model. The RTE (Figs. 3a-c) cannot resolve the finescale pattern due to a lack of cross-phase information. Also, note that the differences seen between the QCM and observations along transect 8 are mostly because of nonlinearity, as confirmed by the comparison to the nonlinear and linearized deterministic model results (the latter is in close agreement with the QCM).
The shortcomings of the RTE are fundamental and a consequence of the fact that it strictly transports variance contributions and omits cross-covariance contributions entirely. In other words, the RTE approximation transports the covariance function $\Gamma$ at zero spatial lag and does not provide information about the correlation (or cross-phase information) between two points separated in space. To illustrate what this implies, and how this information is available in the QC model, we consider the covariance function $\tilde{\Gamma}_{\mathbf{x}}$ at a point $\mathbf{x}_{A}=(5,0) \mathrm{m}$ along the centerline behind the shoal (Figs. 3d-f). Since the incident wave field is (nearly) monochromatic and unidirectional, the resulting covariance function resembles the pattern that would qualitatively be expected from a snapshot of the free surface (Fig. 3d). It is important to note that the 

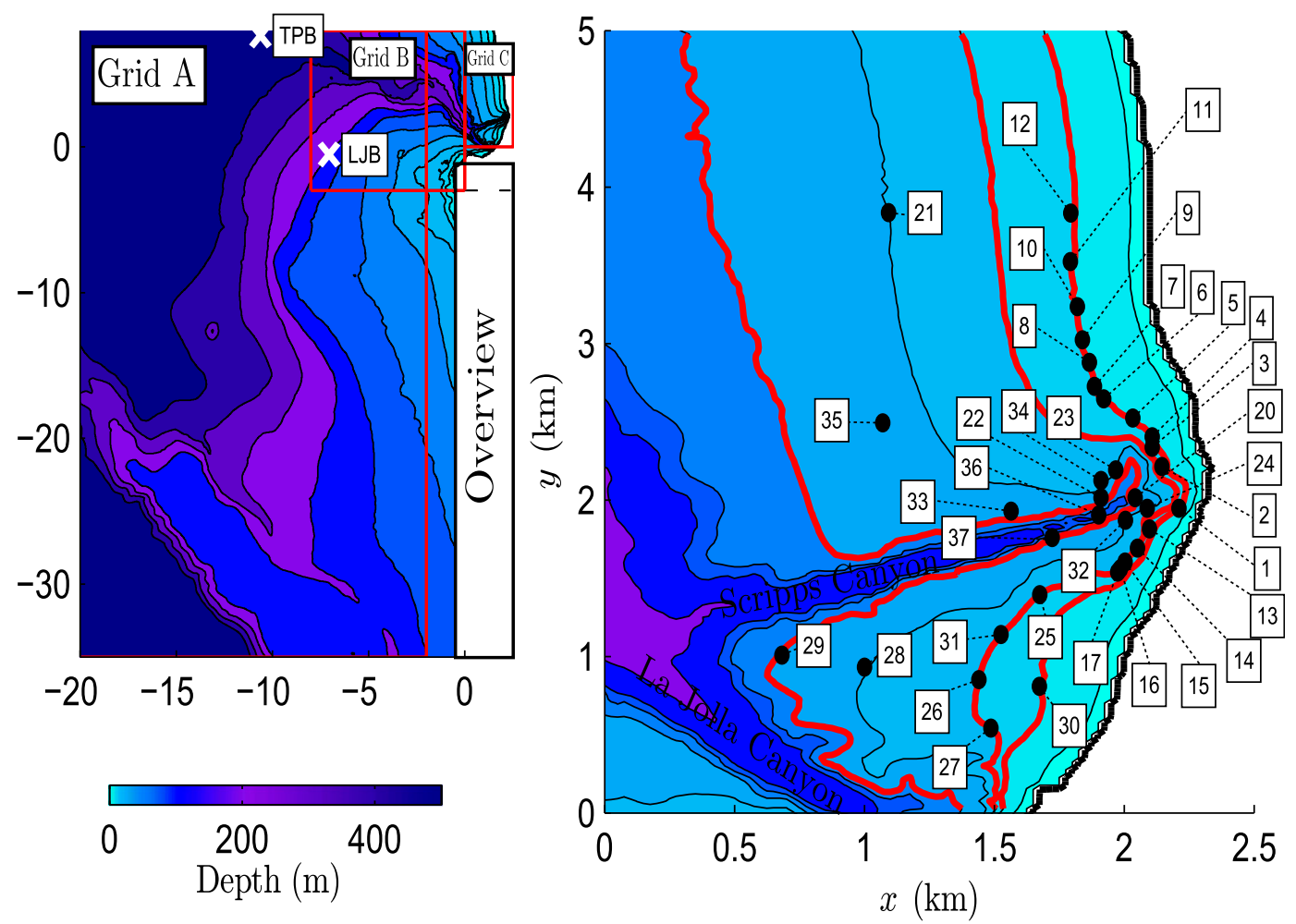

FIG. 4. Bathymetry near (right) Scripps and La Jolla Canyon and the (left) surrounding area. The numbered black dots indicate the instrument locations during the NCEX experiment. Red contour lines correspond to depths of 10,15 and $50 \mathrm{~m}$. The origin is located at $32^{\circ} 49.7^{\prime} \mathrm{N}$ and $117^{\circ} 21.9^{\prime} \mathrm{W}$, with the $x$ and $y$ axes pointing positive east and north, respectively.

QC is fully stochastic, and there is no information on individual realizations of the free surface. The phases in the surface plot are coherent cross phases, and the wavy pattern is a covariance function that does not contain absolute phase information.

Upwave from the shoal, the spatial covariance function takes on the form of a long-crested wave field, with lines of equal phase (or more precisely, equal phase difference) that are alternately in phase at the maxima (or "wave crests") and out of phase at the minima (or "wave troughs") with the wave field at the point $\mathbf{x}_{A}$ considered. Downwave from the shoal, a lateral standing wave pattern emerges, where nodal lines run nearly parallel to the principal propagation direction and points on the opposite direction of the nodal lines are negatively correlated. This is indicative of coherent wave interference, and the pattern closely resembles the classical refraction-diffraction pattern of surface waves in a focal zone. The same cross-correlation function can be estimated from the SWASH and RTE models (see Figs. 3e,f). The covariance function estimated from the SWASH model is practically identical to the QC model (as we would expect it to be), in particular in a region within the coherent radius $\xi_{c} / 4$ (with $\xi_{c}=2 \pi / \Delta k_{\text {spec }}$ ) centered around point $\mathbf{x}_{A}$ (see Fig. 3). Outside this region, the
QCM shows small differences with the SWASH result, in part because at larger spatial lags the approximation implied by the QCM deteriorates (because we are trying to model a monochromatic field) and in part because sidewall reflections (e.g., along $\mathbf{y}^{\prime}=10 \mathrm{~m}$ ) are not accounted for in the QCM. In contrast, the covariance function estimated from the RTE is quite different from the SWASH (and QCM) results (cf. Figs. 3d,e and 3f). The fact that the RTE assumes that the wave field decorrelates much faster than the medium variations, which implies the complete loss of coherent phase information, prevents this model from resolving the structure of the wave field statistics in a focal zone. In regions where waves refract and develop coherent interferences, the QC approximation captures the structure of the spatial covariance function and thus the complete second-order statistics.

\section{Swell over submarine canyons}

Just offshore and to the north of San Diego, stretching from Black's Beach down to La Jolla point (see Fig. 4), the seafloor bathymetry is characterized by two steep submarine canyons: Scripps Canyon (approximately $150 \mathrm{~m}$ deep and $250 \mathrm{~m}$ wide) and La Jolla Canyon (approximately $120 \mathrm{~m}$ deep and $350 \mathrm{~m}$ wide). Along these canyons (see Fig. 4), which extend to $200 \mathrm{~m}$ from shore, 
strong wave refraction occurs because of the steep slopes along the canyon walls (locally exceeding $45^{\circ}$ ). Especially for long-period swell waves, refraction causes extreme spatial gradients in wave height, and locally, coherent interference effects associated with waves arriving along different ray paths are expected to be important (e.g., Magne et al. 2007).

The Nearshore Canyon Experiment (NCEX) was conducted in the fall of 2003 to study wave transformation over the canyons (Thomson et al. 2005, 2007; Magne et al. 2007), with a particular focus on Scripps Canyon. Pressure sensors (locations 13-17 and 20-31), Waverider directional buoys (locations 21 and 32-37), and NORTEK vector current meters (PUVs, location 1-12) were deployed around the canyons (see Fig. $4 \mathrm{~b}$ for locations). The offshore wave conditions were recorded by the permanently deployed Torrey Pines Outer directional Waverider buoy (TPB hereafter) that is located approximately $12.5 \mathrm{~km}$ offshore at $549-\mathrm{m}$ depth (see Fig. 4). The La Jolla Outer Buoy (LJB) is located directly to the west of the NCEX area, but it is situated in relatively shallow water $(200 \mathrm{~m})$ and near a steep slope so that the wave field recorded at this location is generally not suited as an offshore boundary condition.

At all pressure sensor and PUV sites, surface height variance density spectra are obtained from the detrended 3-h pressure records. Each record is subdivided in windowed segments with $50 \%$ overlap and ensemble averaging of the resulting periodograms yields estimates
TABLE 1. Significant wave height $H_{m_{0}}$, peak period $T_{p}$, mean direction $\theta_{\text {mean }}$, and mean directional spreading $\Delta \theta$ at the Torrey Pines Outer Buoy for the swell cases considered.

\begin{tabular}{lccccr}
\hline \hline Case & Date/time (UTC) & $H_{m_{0}}(\mathrm{~m})$ & $T_{p}(\mathrm{~s})$ & $\theta_{\text {mean }}\left(^{\circ}\right)$ & $\Delta \theta\left(^{\circ}\right)$ \\
\hline I & $0000: 0028$ Oct & 0.81 & 16 & 73 & 11 \\
II & $2030: 0016$ Nov & 0.45 & 18.2 & 79 & 7 \\
III & $1530: 0030$ Nov & 0.77 & 15.2 & 16 & 11 \\
\hline
\end{tabular}

of the bottom pressure spectrum with 120 degrees of freedom and frequency resolution $\Delta f=0.0025 \mathrm{~Hz}$. Subsequently, the bandpassed $(0.025-0.2 \mathrm{~Hz})$ freesurface spectrum is obtained using a transfer function from linear theory. For the wave buoys, the spectrum is estimated by averaging five spectra, each obtained from 26-min-long records, with $\Delta f=0.0025 \mathrm{~Hz}$, yielding approximately 120 degrees of freedom. The directional spectrum, needed to force the models at the offshore boundary, is estimated from the measured first four directional Fourier moments using a maximum entropy method (Lygre and Krogstad 1986).

From the 3-month field campaign, we selected three cases to compare the QCM and RTE with observed wave conditions. Because coherent effects are most dominant for directionally narrow fields (cf. Fig. 5 in SJ13), we consider cases where clearly distinguishable swell waves were observed at the TPB, incident from either the south (cases I and II) or from the west (case III). For each case, the bulk parameters are summarized in Table 1 with spectra shown in Fig. 5. Because we focus

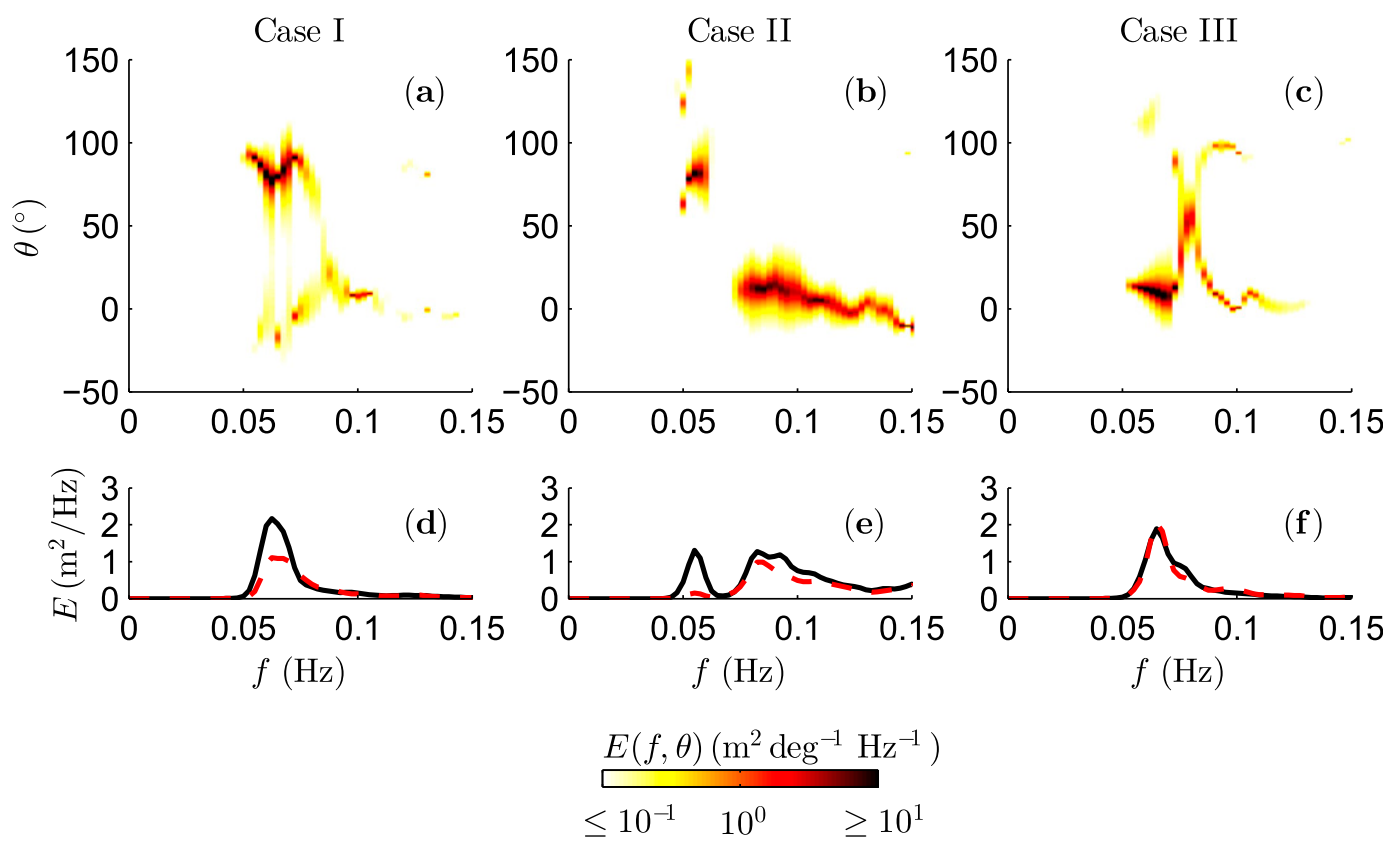

FIG. 5. (top) Directional wave spectra and (bottom) integrated frequency spectra as observed at the TPB in the fall of 2003 for (a), (d) case I, (b), (e) case II, and (c),(f) case III. The red dashed line corresponds to the observed spectra at the LJB. 
(a) Case I

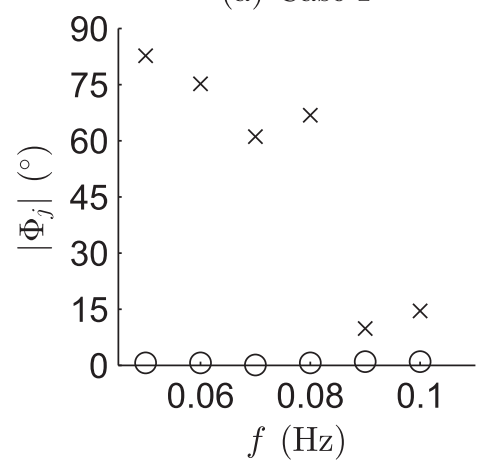

(b) Case II

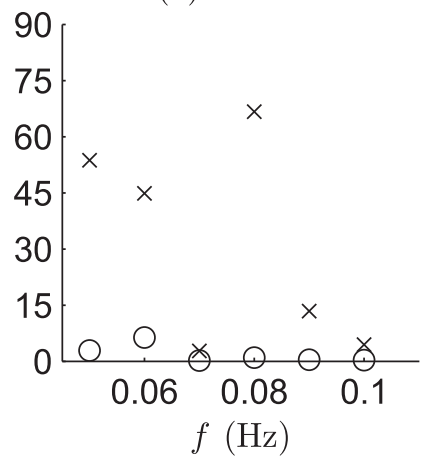

(c) Case III

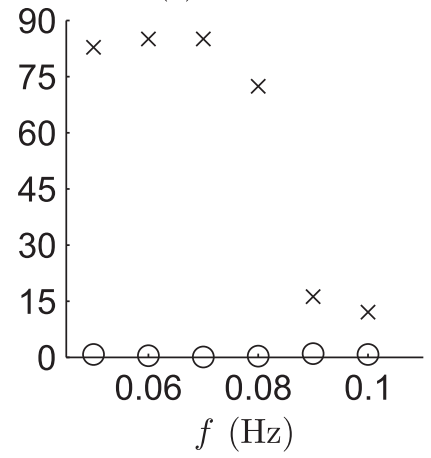

FIG. 6. Absolute phase differences between surface elevation and the velocity components in the dominant wave direction ( $\left|\Phi_{1}\right|$, o markers; the lateral direction $\left|\Phi_{2}\right|, \mathrm{x}$ markers) estimated for different frequency bins in cases I, II, and III at instrument location 3.

on swell, the mixed sea swell system, incident from the west, as present in case II (separated from the distinct southern swell peak; see Fig. 5), was discarded in the present analysis by a low-pass filter $(f<0.07 \mathrm{~Hz}$. $)$. Moreover, all spectra, including observed (cross) spectra, were high-pass filtered $(f>0.025 \mathrm{~Hz})$ to remove infragravity contributions, which are the result of nonlinear interactions and cannot be represented in the present linear model.

To identify the presence of coherent effects that measurably influence wave statistics near the canyon head, we consider the observed swellband cross-covariance spectrum $\boldsymbol{C}_{\eta, \mathbf{u}}(f)=\left(C_{\eta, u_{1}}, C_{\eta, u_{2}}\right)$ between the free surface and the velocity component $u_{1}, u_{2}$ at the PUVs (sites 1-12), which we write as

$C_{\eta, u_{j}}^{f}=\left|C_{\eta, u_{j}}^{f}\right| \exp \left(\mathrm{i} \Phi_{j}^{f}\right)=\int_{f-\Delta f / 2}^{f+\Delta f / 2} C_{\eta, u_{j}}\left(f^{\prime}\right) \mathrm{df}^{\prime}$.

Here, $\left|C_{\eta, u_{j}}\right|$ is the coherence and $\Phi_{j}^{f}$ corresponds to the average phase difference between $u_{j}$ and surface elevation $\eta$ at frequency $f$. Anticipating that interference patterns due to crossing waves propagating toward shore create a nodal structure (partially standing waves) approximately perpendicular to the mean wave direction, the coordinate system is rotated such that the principal component $u_{1}$ is aligned with the mean wave direction $\theta_{\text {mean }}$, and $u_{2}$ is aligned with the lateral direction. Here, the mean direction is defined as $\theta_{\text {mean }}=\operatorname{atan}\left(C_{\eta, v_{2}}^{\text {sum }} / C_{\eta, v_{1}}^{\text {sum }}\right)$ with $C_{\eta, v_{j}}^{\text {sum }}=\sum_{f} \Re\left(C_{\eta, v_{j}}^{f}\right)$, where $v$ denotes the observed particle velocities at the PUVs in the original coordinates, and $\Re$ ( ) denotes the real part of the complex argument considered.

Near Scripps Canyon (location 3), the particle velocities $u_{1}$ are nearly in phase with the surface elevation at each frequency $\left(\left|\Phi_{1}^{f}\right| \approx 0\right.$; Fig. 6), indicative of the predominantly progressive character of the waves in the mean wave direction. However, at low frequencies $(0.05 \leq f \leq 0.08)$, large phase differences (approaching $90^{\circ}$ ) occur, indicative of purely standing wave motion. This clearly indicates a (partially) standing wave structure in the lateral direction at these frequencies, which is associated with coherent interference of the surface waves. These large phase differences for $\left|\Phi_{2}^{f}\right|$ are only seen near the canyon head (locations 1-6), which is consistent with the refraction-induced wave interference expected in this region. For locations farther away from the canyon head (e.g., locations 7-12), $\left|\Phi_{2}^{f}\right|$ is generally small (not shown). The observations thus show that the coherent effects affect the wave statistics near the canyon heads. Although the variance levels of the wave motion in the lateral direction is small, these standing modes can develop a coherent nodal structure and drive subtle alongshore variations of wave orbital velocity statistics near the shore that are important to wave-induced circulation, transport processes, and coastal morphodynamics.

\section{a. Model setup}

The spectral models are numerically evaluated on a set of nested rectangular spatial grids (see Table 2).

TABLE 2. Model parameters used for the different nested grids A to C. The spectral resolution was set to $\Delta k=0.01 \mathrm{rad} \mathrm{\textrm {m } ^ { - 1 }}$ in all cases.

\begin{tabular}{cccccccrrr}
\hline \hline Grid & $x(\mathrm{~km})$ & $y(\mathrm{~km})$ & $\Delta x(\mathrm{~m})$ & $N_{x}$ & $N_{y}$ & $k_{x}\left(\mathrm{rad} \mathrm{m}^{-1}\right)$ & $k_{y}\left(\mathrm{rad} \mathrm{m}^{-1}\right)$ & $M_{x}$ & $M_{y}$ \\
\hline A & -17 & -36 & 50 & 300 & 880 & -0.0205 & -0.0605 & 121 \\
B & -8 & -2 & 25 & 120 & 360 & -0.0205 & -0.1005 & 121 \\
C & 0 & 0 & 25 & 100 & 200 & -0.0205 & -0.1505 & 171 & 301 \\
\hline
\end{tabular}



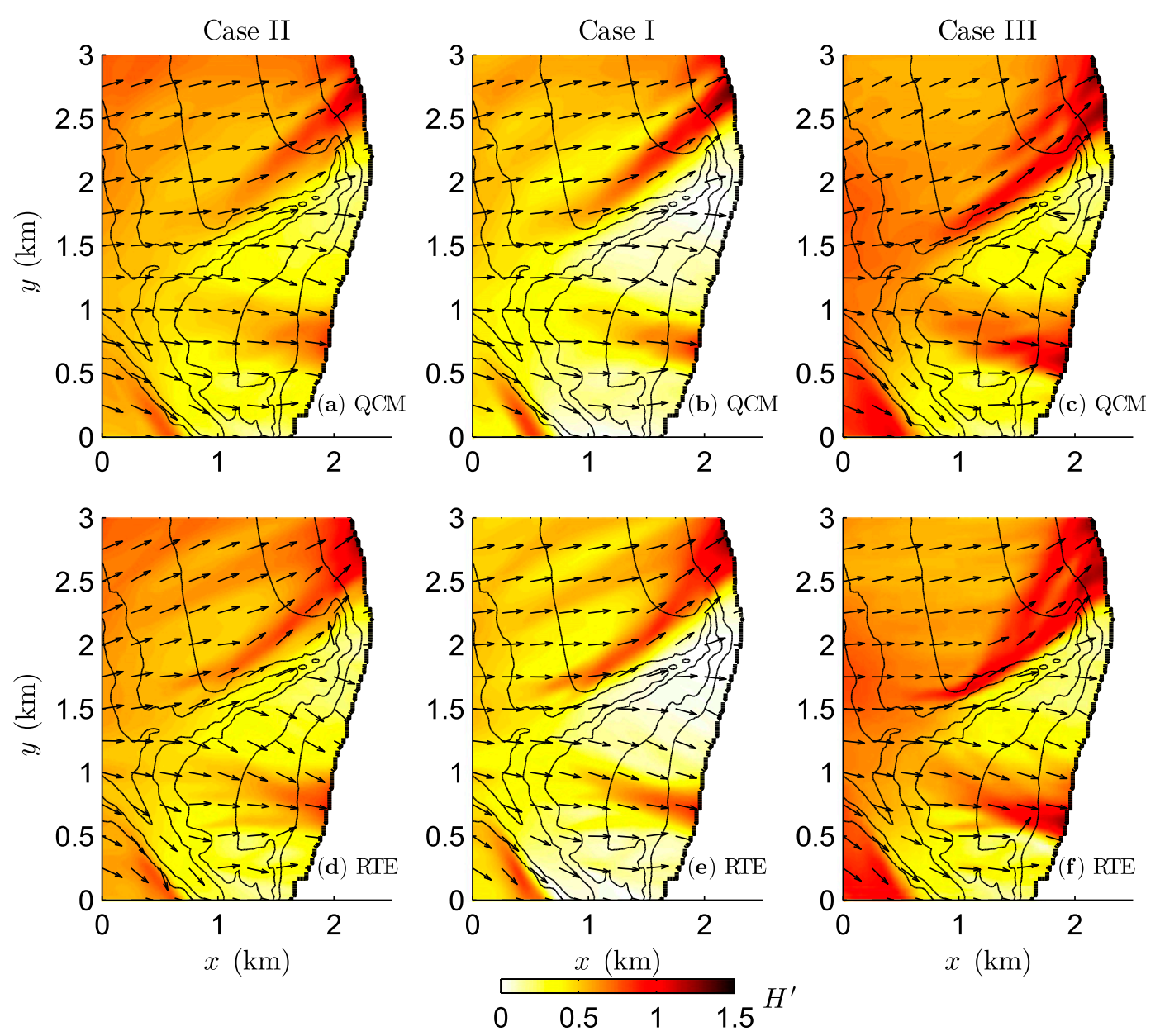

FIG. 7. Wave heights (normalized with the incident wave height) predicted over the canyons for (a),(d) case I, (b),(e) case II, and (c),(f) case III by the (top) QCM and (bottom) RTE. Superimposed arrows correspond to the mean wave direction.

The coarsest grid (A) is forced by directional spectra derived from the buoy data on the western boundary (Fig. 4), whereas on the southern and northern boundaries $\partial_{y} \mathscr{E}(\mathbf{k}, \mathbf{x})=0$ is maintained for components directed into the domain. The finest grid $(\mathrm{C})$ is used near the NCEX site in the area depicted in Fig. 4 (right). Moreover, going from $\mathrm{A}$ to $\mathrm{C}$, the spectral domain is successively enlarged to ensure that the wave spectrum falls within the computational domain. On the outer grid A, where, owing to deep-water conditions, the wave field is effectively homogeneous, computations were done exclusively with the RTE. For the other two domains, results were obtained using both the RTE and the QCM.

For southerly waves (cases I and II), the boundary derived from the TPB is not optimal. For these cases, waves that arrive at the NCEX site are refracted over the continental shelf well south of the TPB, whereas southerly waves that arrive at the TPB do not reach the
NCEX site. These effects are seen in the large differences between the swell peaks observed at the TPB and LJB for southerly swells (see case I and II in Fig. 5). Nevertheless, the ratio $R$ between the predicted to measured significant wave heights at the LJB was near unity for cases II $(R=1.01)$ and III $(R=0.94)$, and only for case I did the ratio differ significantly $(R=0.74)$. For the latter case the spectra at the boundary were rescaled with $1 / R^{2}$ to obtain more realistic conditions at the LJB and presumably the NCEX area.

\section{b. Results}

For the southerly swell cases (I and II), a significant part of the energy is refracted toward the coast before it arrives at the NCEX site because of the relatively shallow region south of the canyons (see Fig. 4). Hence, wave energy is already much reduced when it arrives at the NCEX site (Figs. 7a,b). The waves subsequently refract strongly over the steep canyon walls, which is 

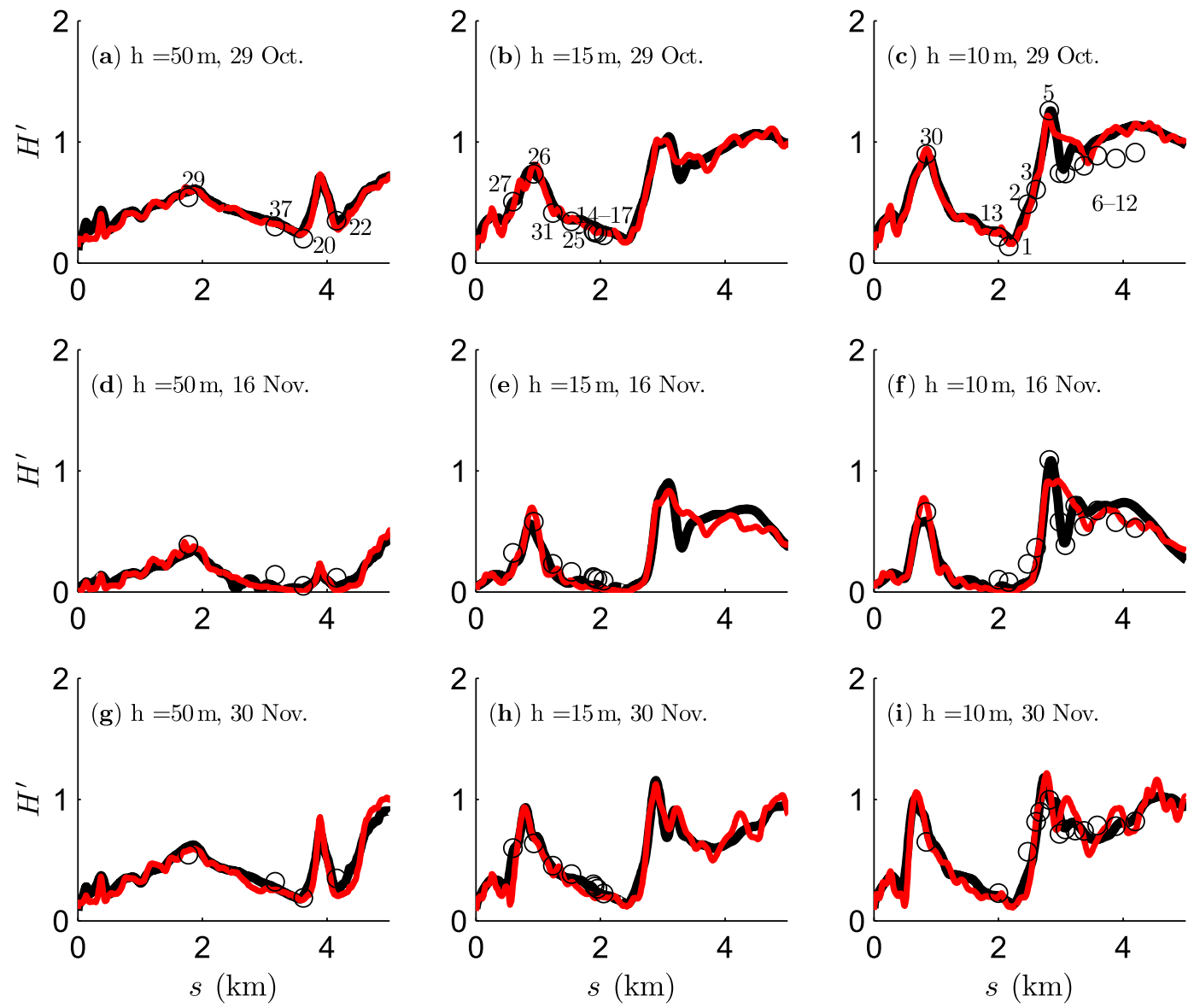

FIG. 8. Normalized wave height $H^{\prime}=H_{m 0} / H_{m 0}^{\mathrm{TPB}}$ along the indicated depth contours (Fig. 4) for (a)-(c) case I, (d)-(f) case II, and (g)-(i) case III. Observations (open circles) are compared to results from the RTE (red dashed line) and the QCM (black solid line).

visible in the bands of enhanced wave height along the canyon walls. In between the canyons, the convex shape of the topography focuses wave energy so that a mild focal zone emerges. For westerly swells, the pattern is again dominated by the local geometry of the canyons, with a band of enhanced wave energy along the canyon walls and a mild focal region in between the two canyons (Figs. 7c,d).

Although the QCM and RTE predictions appear to be fairly similar, there are some important differences. To intercompare the models in more detail, and compare simulation results from both models with observations, we consider transects of wave height estimates along different depth contours (Fig. 8). Overall correspondence between the observations and both models is reasonable, specifically for the 50- and 15-m contour lines. However, along the $10-\mathrm{m}$ contour line (around $s=2.5 \mathrm{~km}$, where $s$ represents the along-contour distance measured from the starting point on the north edge of the NCEX area), significant differences between the models are seen. It is in this region that interference occurs between waves that travel in a western direction and waves that are refracted out of the canyon. This results in rapid oscillations of the wave heights, which is particularly visible in the observations for case II. The QCM reproduces this oscillatory behavior almost perfectly (at least near $s=3 \mathrm{~km}$ ), whereas the variations in the RTE model are much less extreme and do not capture the rapid changes in the mean wave heights.

As highlighted in the analysis of the laboratory data, the QCM captures the complete second-order statistics and thus inherently contains spatial information of coherent patterns and standing wave fields. To analyze the wave pattern surrounding the canyon heads, we consider the covariance function as calculated by the QCM at site 31 on the northern edge of Scripps Canyon and at site 24 in the focal area between the two canyons (Fig. 9). For illustrative purposes, we include a few ray trajectories that are initiated at $x=0$ using the predicted mean wavenumber $\tilde{\mathbf{k}}\left(=\int \mathbf{k} \mathscr{E} d \mathbf{k} / \mathscr{\mathscr { V }}\right)$ to help interpretation of the results.

In the mean wave direction (approximately aligned with the wave rays), the covariance function attains its 

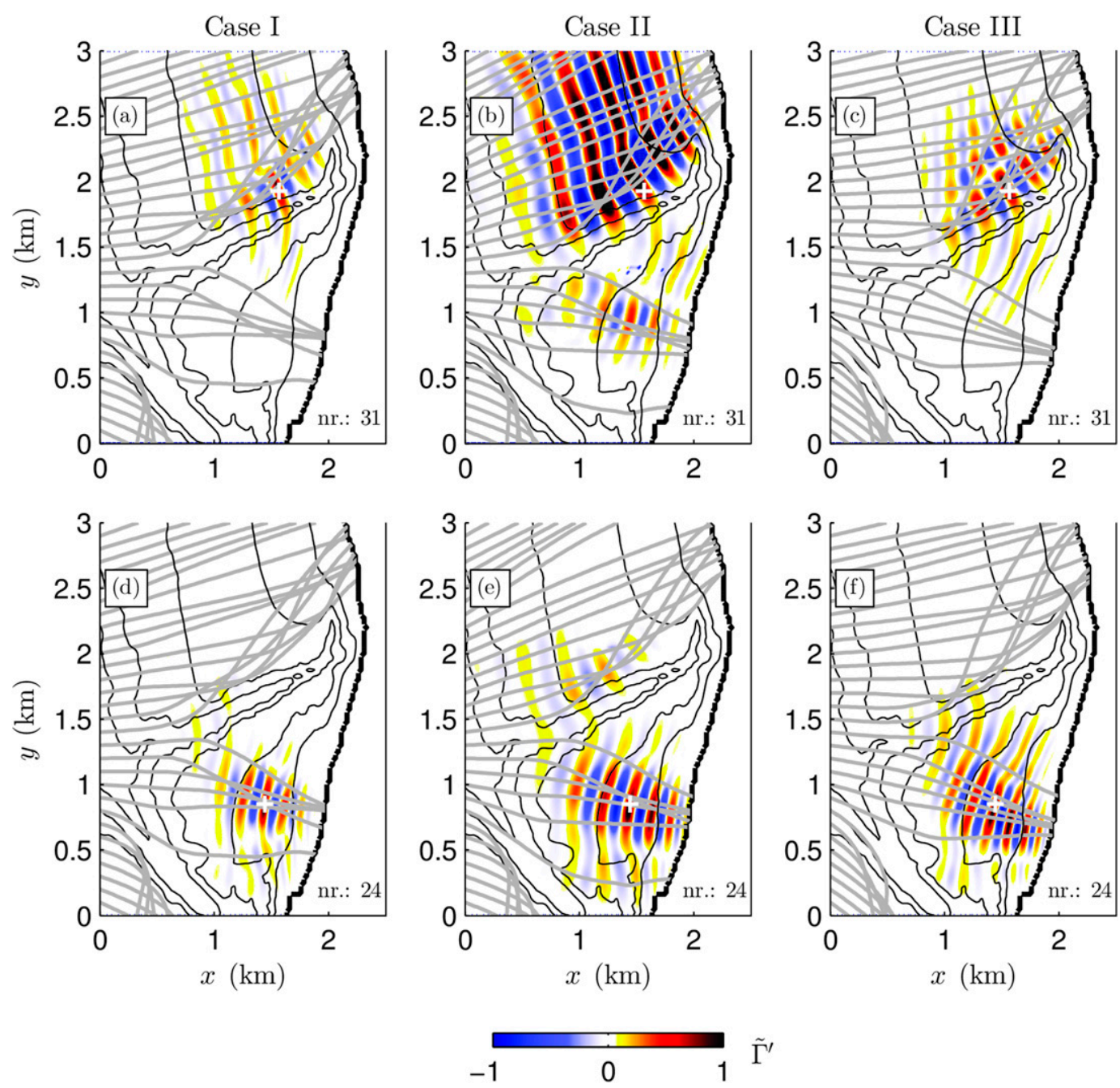

FIG. 9. Normalized covariance functions $\tilde{\Gamma}_{\mathbf{x}}^{\prime}\left[=\tilde{\Gamma}_{\xi} / \mathscr{\mathscr { C }}(\mathbf{x})\right]$ obtained from the QCM results for (a),(d) case I, (b),(e) case II, and (c),(f) case III at sites (top) 31 and (bottom) 24 with superimposed bottom contours (thin black lines) and a few representative ray paths of the dominant swells (thick gray lines). The sites considered in each panel (located at the white crosses) are indicated by the number in the lower-right corner.

typical oscillatory pattern, indicative of predominantly propagating wave motion. However, near site 31 (Figs. 9a-c), the covariance function shows a nodal structure in the lateral direction, which implies fast variations of the statistics associated with crossing waves (as also indicated by the crossing rays in this region). In case III (Fig. 9c), a clear nodal pattern emerges where in the lateral (or along crest) direction the covariance function alternates between positive and negative values. The covariance function centered at site 24 retains a structure more in line with the assumptions of quasi-homogeneous theory, with a modulated oscillatory structure in the wave direction (the limited extend of the correlation function due to the finite width of the spectrum) and a slowly decaying correlation with constant phase difference in the lateral direction (indicative of the finite directional width of the wave field). This is consistent with the observation that quasi-homogeneous theory (RTE) and quasi-coherent theory (QCM) predict similar wave heights in this region. In fact, the covariance functions predicted by the RTE are similar to those of the QCM near site 24, although they decay more rapidly (not shown).

From the covariance functions, we see that because of the strong refraction by the canyons, the waves just north and south of Scripps Canyon are statistically nearly independent in cases I and III. That is, the dominant swells, traveling almost parallel to the canyon axis, are trapped on the north side of the canyon, and a weak, uncorrelated component that crosses the canyon from more oblique angles dominates the wave motion south of the canyon. For case II, the wave field is so narrow that some correlation between waves on opposite sides of the Scripps Canyon remains. The directional narrowness of the incident waves 
during case II is also apparent from the large spatial extent of the correlation bands just north of the canyon.

\section{Conclusions}

In this study, we developed a stochastic modeling framework for describing the effects of coherent wave interference on spectral wave evolution. By considering a finite coherent footprint of the wave field, the quasicoherent theory of SJ13 was reformulated in a form similar to a radiative transfer equation, commonly used in operational wave prediction models, but with an additional source term to account for the coherent effects in the wave evolution. The transported variable in this equation is a coupled-mode spectrum, essentially a generalization of the variance density spectrum, which allows for the evolution of cross-phase information and thus the complete spatial covariance function. We verified that the model captures the complete second-order statistics, including mean wave heights and the wave covariance function, for coherently interfering waves by comparison of model results to laboratory observations of a wave focal zone behind a submerged shoal and with Monte Carlo simulations with a deterministic model. Comparison of model predictions to field observations obtained during the Nearshore Canyon Experiment (NCEX) at Scripps Canyon, a submarine canyon on the southern California coast, demonstrates the improved predictive capability of the new stochastic model. In particular, the QCM accurately predicts the observed interference patterns of crossing waves (near the canyon head) that are neglected in traditional models based on the radiative transfer equation. These results, and in particular the comparison to field observations, show that the QCM can resolve finescale structures in nearshore wave statistics associated with crossing wave fields, which contributes to our understanding of these dynamics near the coast and improves the ability to model nearshore wave statistics, wave-driven circulation, and transport processes near the coast.

Acknowledgments. This research is supported by the U.S. Office of Naval Research (Littoral Geosciences and Optics Program and Physical Oceanography Program) and by the National Oceanographic Partnership Program. We thank M. Zijlema, who supplied the initial version of the BiCGSTAB solver used in the present work; A. Reniers, who provided financial support for PBS; and L. Holthuijsen, for continued support of PBS.

\section{APPENDIX A}

\section{Fourier Transform Operators}

As we make frequent use of continuous and discrete Fourier transforms, it is convenient to introduce the following operators. Let $\zeta$ be a dummy continuous function, for which we denote the conjugate pair as $\zeta(\mathbf{x}), \hat{\zeta}(\mathbf{k})$, such that $\hat{\zeta}(\mathbf{k})=\mathscr{F}_{\mathbf{x}, \mathbf{k}}[\zeta(\mathbf{x})]$ and $\left.\zeta(\mathbf{x})=\mathscr{F}_{\mathbf{k}, \mathbf{x}}^{-1} \hat{\zeta}(\mathbf{k})\right]$, with

$$
\begin{aligned}
& \mathscr{F}_{\mathbf{x}, \mathbf{k}}[\zeta(\mathbf{x})]=\frac{1}{(2 \pi)^{2}} \int \zeta(\mathbf{x}) \exp (-\mathrm{ik} \cdot \mathbf{x}) d \mathbf{x}, \quad \text { and } \\
& \mathscr{F}_{\mathbf{k}, \mathbf{x}}^{-1}[\hat{\zeta}(\mathbf{k})]=\int \hat{\zeta}(\mathbf{k}) \exp (\mathbf{i k} \cdot \mathbf{x}) d \mathbf{k},
\end{aligned}
$$

and the transforms are assumed to exist in the context of generalized functions (Strichartz 1993).

In a similar fashion, we define the Fourier transform pair for a periodic function $\zeta(\mathbf{x})$ with period $L$ for a discrete set of wavenumbers $\mathbf{k}_{m_{1}, m_{2}}=\left(m_{1}, m_{2}\right) \Delta k$ (with $\Delta k=2 \pi / L$ and $m_{1}, m_{2}$ integers) as $\zeta(\mathbf{x}), \hat{\zeta}_{k}$, where

$$
\begin{aligned}
& \hat{\zeta}^{\mathbf{k}}=\overline{\mathscr{F}}_{\mathbf{x}, \mathbf{k}}[\zeta(\mathbf{x})]=\frac{1}{L^{2}} \int_{-L / 2}^{L / 2} \zeta(\mathbf{x}) \exp (-\mathrm{i} \mathbf{k} \cdot \mathbf{x}) d \mathbf{x}, \\
& \zeta(\mathbf{x})=\overline{\mathscr{F}}_{\mathbf{k}, \mathbf{x}}^{-1}\left\{\hat{\zeta}^{\mathbf{k}}\right\}=\sum_{\mathbf{k}} \hat{\zeta}^{\mathbf{k}} \exp (\mathrm{ik} \cdot \mathbf{x}) .
\end{aligned}
$$

\section{APPENDIX B}

\section{Discrete Model}

To consider the numerical solution of the QC approximation, we consider the solution on a discrete regular rectangular mesh in both geographical and wavenumber space. For the spatial and wavenumber mesh we set

$$
\begin{aligned}
\mathbf{x}_{m_{1}^{x}, m_{2}^{x}} & =\mathbf{x}_{0}+\left(m_{1}^{x} \Delta x_{1}, m_{2}^{x} \Delta x_{2}\right), \\
\mathbf{k}_{m_{1}^{k}, m_{2}^{k}} & =\mathbf{k}_{0}+\left(m_{1}^{k} \Delta k_{1}, m_{2}^{k} \Delta k_{2}\right),
\end{aligned}
$$

with $m_{j}^{x} \in\left\{0 \ldots M_{j}^{x}\right\}, m_{j}^{k} \in\left\{0 \ldots M_{j}^{k}\right\}$, and where $\mathbf{x}_{0}$ and $\mathbf{k}_{0}$ denote the coordinates of the lower-left corner of the geographical and wavenumber grid, respectively. For brevity, we denote the discrete coupled-mode spectrum evaluated at $\mathbf{x}_{m_{1}^{x}, m_{2}^{x}}, \mathbf{k}_{m_{1}^{k}, m_{2}^{k}}$ as $\mathscr{E}^{\mathbf{k} \mathbf{x}}(t)$, where the dependency on the subscripts $m_{j}^{x}, m_{j}^{k}$ is implied. With these definitions in place, the spatially discrete and stationary version of the quasi-coherent approximation can be expressed as

$$
\begin{aligned}
\mathbf{c}_{k} \cdot \mathscr{D}_{\mathbf{k}} \mathscr{E}^{\mathbf{k} \mathbf{x}}+\mathbf{c}_{x} \cdot \mathscr{D}_{\mathbf{x}} \mathscr{E}^{\mathbf{k} \mathbf{x}} \\
\quad=-\mathrm{i} \sum_{|\mathbf{q}| \leq q_{\max }} \Delta \hat{\Omega}^{\mathbf{q}}\left(\mathbf{k}, \mathbf{x},-\mathrm{i} \mathscr{D}_{\mathbf{x}}\right) \mathscr{E}^{\mathbf{k}-\mathbf{q} / 2, \mathbf{x}}+*
\end{aligned}
$$


Here, $\mathscr{D}_{\mathbf{x}}$ and $\mathscr{D}_{\mathbf{k}}$ denote linear finite-difference operators that approximate $\boldsymbol{\nabla}_{\mathbf{x}}$ or $\boldsymbol{\nabla}_{\mathbf{k}}$, respectively, by means of second-order upwind approximations. The operator $\mathscr{D}_{\mathbf{x}} \mathcal{E}^{\mathbf{k} \mathbf{x}}$ is defined as

$\mathscr{D}_{x_{j}} \mathscr{E}^{\mathbf{k} \mathbf{x}}=s_{j} \frac{3 \mathscr{E}^{\mathbf{k} \mathbf{x}}-4 \mathscr{E}^{\mathbf{k} \mathbf{x}-\Delta \tilde{\mathbf{x}}^{j}}+\mathscr{E}^{\mathbf{k} \mathbf{x}-2 \Delta \tilde{\mathbf{x}}}}{2 \Delta x_{j}}$,

with $\mathbf{s}=\operatorname{sgn}(\mathbf{k})$ and $\Delta \tilde{\mathbf{x}}^{j}=s_{j}\left(\delta_{1 j} \Delta x_{1}, \delta_{2 j} \Delta x_{2}\right)$, where $\delta_{i j}$ denotes the Kronecker delta. The operator $\mathscr{D}^{\mathbf{k}} \mathscr{E}_{\mathbf{k}, \mathbf{x}}$ is defined analogously, but then along the spectral dimensions

$\mathscr{D}_{k_{j}} \mathscr{C}^{\mathbf{k} \mathbf{x}}=\tilde{s}_{j} \frac{3 \mathscr{E}^{\mathbf{k} \mathbf{x}}-4 \mathscr{E}^{\mathbf{k}-\Delta \tilde{\mathbf{k}}^{j}, \mathbf{x}}+\mathscr{E}^{\mathbf{k}-2 \Delta \tilde{\mathbf{k}}^{j}, \mathbf{x}}}{2 \Delta k_{j}}$,

where $\tilde{\mathbf{s}}=\operatorname{sgn}\left(\mathbf{c}_{x}\right)$ and $\Delta \tilde{\mathbf{k}}^{j}=\tilde{s}_{j}\left(\delta_{1 j} \Delta k_{1}, \delta_{2 j} \Delta k_{2}\right)$. Upwind approximations are used to enhance convergence in the iterative solution technique used to solve the system of equations in a manner similar to Booij et al. (1999); we discuss this in more detail in what follows.

To exclude interactions between waves and topographical variations on the infrawave scale, which are excluded at the order $O(\varepsilon)$ considered, the sum over $\mathbf{q}$ is restricted to $|\mathbf{q}| \leq q_{\max }$. Here, $q_{\max }$ is the minimum of $|\mathbf{k}| / 2$ or a predescribed maximum bottom wavenumber component. When solving for the RTE, we disregard this sum altogether.

At the geographic boundary (along the lines $m_{1}^{x}=0, m_{1}^{x}=M_{1}^{x}$ and $m_{2}^{x}=0, m_{2}^{x}=M_{2}^{x}$ ), the wave spectrum is prescribed for wavenumbers directed into the computational domain. For the spectral boundary (along the lines $m_{1}^{k}=0, m_{1}^{k}=M_{1}^{k}$ and $m_{2}^{k}=0, m_{2}^{k}=M_{2}^{k}$ ), we assume that it is located at wavenumbers that are effectively deep-water waves, so that the interaction with components outside the computational domain (assumed to be zero) can be neglected. Moreover, at points adjacent to the geographic or spectral boundary (e.g., along the line $m_{1}^{x}=1$ ) first-order approximations are used if Eqs. (B3) or (B4) reference points outside the computational domain.
Here, the discrete operators $\mathscr{D}_{x_{j}}$ and $\mathscr{D}_{k_{j}}$ are locally reduced to

$$
\begin{gathered}
\mathscr{D}_{x_{j}} \mathscr{E}^{\mathbf{k} \mathbf{x}}=s_{j} \frac{\mathscr{E}^{\mathbf{k} \mathbf{x}}-\mathscr{E}^{\mathbf{k} \mathbf{x}-\Delta \tilde{\mathbf{x}}^{j}}}{\Delta x_{j}}, \\
\mathscr{D}_{k_{j}} \mathscr{C}^{\mathbf{k} \mathbf{x}}=\tilde{s}_{j} \frac{\mathscr{C}^{\mathbf{k} \mathbf{x}}-\mathscr{E}^{\mathbf{k}-\Delta \tilde{\mathbf{k}}^{j} \mathbf{x}}}{\Delta k_{j}},
\end{gathered}
$$

respectively.

\section{a. Coefficients}

The geographic and spectral propagation velocities are defined as $\mathbf{c}_{k}^{\mathrm{RTE}}(\mathbf{k}, \mathbf{x})=-\sigma_{h} \nabla_{\boldsymbol{x}} h$ and $\mathbf{c}_{x}^{\mathrm{RTE}}=\tilde{\mathbf{k}} \sigma_{k}$, whereas the difference between $\Omega$ and the plane approximation $\Omega_{\mathrm{RTE}}$ [see Eq. (12)] is used to calculate $\Delta \hat{\Omega}$. To calculate these, we define a local geographic grid $\mathbf{x}^{\prime}$ over the coherent footprint centered at $\mathbf{x}$, and its conjugate set of wavenumbers $\mathbf{q}$ as

$\mathbf{x}_{m_{1}^{x^{\prime}}, m_{2}^{x^{\prime}}}^{\prime}=\left(m_{1}^{x^{\prime}} \Delta x_{1}, m_{2}^{x^{\prime}} \Delta x_{2}\right), \quad \mathbf{q}_{m_{1}^{q}, m_{2}^{q}}=\left(m_{1}^{q} \Delta q_{1}, m_{2}^{q} \Delta q_{2}\right)$,

with $m_{j}^{x^{\prime}}, m_{j}^{q} \in\left\{-M_{j}^{q} / 2 \ldots M_{j}^{q} / 2\right\}, M_{j}^{q}=2 q_{\max } / \Delta q_{j}$, and $\Delta x_{j}=2 \pi\left(M_{j}^{q} \Delta q_{j}\right)^{-1}$. To avoid interpolation, it is convenient to ensure the sum in Eq. (B2) over q coincides with the $\mathbf{k}$ grid, so we set $\Delta q_{j}=2 \Delta k_{j}$, assuming that $\Delta k_{j} \leq 2 \pi / \xi_{c}$. With these definitions in place, the coefficients of the operator $\Delta \hat{\Omega}$ can now be obtained by a discrete Fourier transform. However, to avoid errors due to jump discontinuities between the nonsmoothly matching domain borders in a periodic extension of $\Delta \hat{\Omega}$, we define the transform as

$$
\Delta \hat{\Omega}=\overline{\mathscr{F}}_{\mathbf{x}^{\prime}, \mathbf{q}}\left[W_{1}\left(x_{1}^{\prime}\right) W_{2}\left(x_{2}^{\prime}\right) \Delta \Omega\right],
$$

where $W_{j}\left(x_{j}^{\prime}\right)$ are window functions that smoothly transitions to 0 near the edges of the domain, and for which in the present work we use a tapered cosine (Tukey) window that is given by

$$
W_{j}\left(x_{j}^{\prime}\right)=\left\{\begin{array}{ll}
\frac{1}{2}+\frac{1}{2} \cos \left\{\pi\left[\left(x_{j}^{\prime}-l_{j}+\gamma l_{j}\right) /\left(\gamma l_{j}\right)\right]\right\} & \text { if } \quad x_{j}^{\prime}>(1-\gamma) l_{j} \\
\frac{1}{2}+\frac{1}{2} \cos \left\{\pi\left[x_{j}^{\prime} /\left(\gamma l_{j}\right)-1\right]\right\} & \text { if } \quad x_{j}^{\prime}<\gamma l_{j} \\
1 & \text { elsewhere }
\end{array} .\right.
$$

Here, $l_{j}=2 \pi / \Delta q_{j}$ is the length of the $j$ th side of the $\mathbf{x}^{\prime}$ domain, and $\gamma$ is a dimensionless width parameter controlling the length of the transitional area where $W \rightarrow 0$ and which is set to $\gamma=0.1$. 


\section{b. Iterative solution technique}

The resulting set of equations involves $M=$ $M_{1}^{x} M_{2}^{x} M_{1}^{k} M_{2}^{k}$ variables so that solving for the steady state involves inverting a large sparse $M \times M$ matrix. Solving this system directly is difficult, as even for a moderate number of grid points in each of the four dimensions, the total number of points quickly becomes large. However, because propagation principally occurs in geographic space and is dominated by the LHS of Eq. (B3), the system can be solved iteratively in a marching fashion, similar to the method employed in Booij et al. (1999).

Hereto, $\mathbf{k}$ space is subdivided into four quadrants, each bounded by the Cartesian axes, which we will number 1 till 4 in a counterclockwise fashion, where quadrant 1 is the set $Q_{1}=\left(\mathbf{k}, \mathbf{x} \mid k_{1}>0 \wedge k_{2} \geq 0\right)$. During a single Gauss-Seidel iteration, each of the four quadrants of the spectral domain is visited consecutively using four sweeps per iteration. During each sweep, only points that belong to the quadrant are updated. For example, during iteration step $n$, in the $m$ th sweep $\left(n_{m}\right)$, the quadrant $m$ is considered, and for all points $\mathbf{P}=$ $\left(k_{1}, k_{2}, x_{1}, x_{2}\right)$ where $\mathbf{P} \in Q_{m}$, we substitute the unknown $\mathscr{E}_{\mathbf{P}}^{n_{m}}$ in Eq. (B2) $\forall \mathbf{P}$. Conversely, for all points $\mathbf{R}=\left(k_{1}, k_{2}, x_{1}, x_{2}\right)$ where $\mathbf{R} \notin Q_{m}$ is approximated by the values from the most recent update at $n_{m-1}$ [with $\left.n_{0}=(n-1)_{m}\right]$, and for these we therefore substitute the known values $\mathscr{E}_{\mathbf{R}}^{n_{m-1}}$. Not only does this reduce the number of unknowns per sweep, the structure of the resulting matrix is such that spatial dependencies involving $\mathscr{E}_{\mathbf{P}}^{n_{m}}$ only occur in the downwave direction, and consequently we only need to invert a $M_{1}^{k} M_{2}^{k}$ by $M_{1}^{k} M_{2}^{k}$ matrix involving those wavenumbers $\boldsymbol{k}$ in the quadrant at each spatial point. If the topography is captured with $M_{q}^{2}$ Fourier modes, this gives rise to a dense linear system containing $M_{1}^{q} M_{2}^{q}$ diagonals. However, given that for slowly varying topography the off-diagonal contributions are small, the resulting system can still be solved relatively fast using the BicGStab method with an ILU preconditioner (Van der Vorst 1992).

In the cases considered, the resulting algorithm always converged to a solution within relatively few iterations $(n<10)$, where the solution was considered to be converged after a complete iteration $n_{4}$, when for each spatial point the following criterion was met:

$$
\frac{\sum_{\mathbf{k}}\left(\mathcal{E}_{\mathbf{k}, \mathbf{x}}^{n_{4}}-\mathcal{E}_{\mathbf{k}, \mathbf{x}}^{(n-1)_{4}}\right)^{2}}{\sum_{\mathbf{k}}\left(\mathcal{E}_{\mathbf{k}, \mathbf{x}}^{n_{4}}\right)^{2}}<\alpha^{2}, \quad \forall \mathbf{x} .
$$

Here, $\alpha$ is the convergence criterion, set to $\alpha=10^{-4}$ in the present study.

\section{REFERENCES}

Agnon, Y., and A. Sheremet, 1997: Stochastic nonlinear shoaling of directional spectra. J. Fluid Mech., 345, 79-99, doi:10.1017/ S0022112097006137.

Apotsos, A., B. Raubenheimer, S. Elgar, and R. T. Guza, 2008: Testing and calibrating parametric wave transformation models on natural beaches. Coastal Eng., 55, 224-235, doi:10.1016/ j.coastaleng.2007.10.002.

Ardhuin, F., and T. H. C. Herbers, 2002: Bragg scattering of random surface gravity waves by irregular sea bed topography. J. Fluid Mech., 451, 1-33, doi:10.1017/S0022112001006218.

, W. C. O'Reilly, T. H. C. Herbers, and P. F. Jessen, 2003: Swell transformation across the continental shelf. Part I: Attenuation and directional broadening. J. Phys. Oceanogr., 33, 1921-1939, doi:10.1175/1520-0485(2003)033<1921:STATCS>2.0.CO;2.

Battjes, J. A., and J. P. F. Janssen, 1978: Energy loss and set-up due to breaking of random waves. Proc. 16th Conf. on Coastal Engineering, Hamburg, Germany, ASCE, 569-587.

Berkhoff, J. C. W., N. Booij, and A. C. Radder, 1982: Verification of numerical wave propagation models for simple harmonic linear water waves. Coastal Eng., 6, 255-279, doi:10.1016/ 0378-3839(82)90022-9.

Booij, N., R. C. Ris, and L. H. Holthuijsen, 1999: A third-generation wave model for coastal regions: 1 . Model description and validation. J. Geophys. Res., 104, 7649-7666, doi:10.1029/ 98JC02622.

Dodet, G., X. Bertin, N. Bruneau, A. B. Fortunato, A. Nahon, and A. Roland, 2013: Wave-current interactions in a wavedominated tidal inlet. J. Geophys. Res. Oceans, 118, 15871605, doi:10.1002/jgrc.20146.

Eldeberky, Y., 1996: Nonlinear transformation of wave spectra in the nearshore zone. Ph.D. thesis, Delft University of Technology, $203 \mathrm{pp}$.

Elgar, S., T. H. C. Herbers, and R. T. Guza, 1994: Reflection of ocean surface gravity waves from a natural beach. J. Phys. Oceanogr., 24, 1503-1511, doi:10.1175/1520-0485(1994)024<1503: ROOSGW $>2.0 . \mathrm{CO} ; 2$.

Freilich, M. H., and R. T. Guza, 1984: Nonlinear effects on shoaling surface gravity waves. Philos. Trans. Roy. Soc., A311, 1-41, doi:10.1098/rsta.1984.0019.

Grant, W. D., and O. S. Madsen, 1979: Combined wave and current interaction with a rough bottom. J. Geophys. Res., 84, 17971808, doi:10.1029/JC084iC04p01797.

Hasselmann, K., 1962: On the non-linear energy transfer in a gravity-wave spectrum Part 1 . General theory. J. Fluid Mech., 12, 481-500, doi:10.1017/S0022112062000373.

_ 1974: On the spectral dissipation of ocean waves due to white capping. Bound.-Layer Meteor., 6, 107-127, doi:10.1007/ BF00232479.

Herbers, T. H. C., and M. C. Burton, 1997: Nonlinear shoaling of directionally spread waves on a beach. J. Geophys. Res., 102, 21 101-21 114, doi:10.1029/97JC01581.

Janssen, T. T., T. H. C. Herbers, and J. A. Battjes, 2006: Generalized evolution equations for nonlinear surface gravity waves over two-dimensional topography. J. Fluid Mech., 552, 393418, doi:10.1017/S0022112006008743.

,-- , and,- 2008 : Evolution of ocean wave statistics in shallow water: Refraction and diffraction over seafloor topography. J. Geophys. Res., 113, C03024, doi:10.1029/2007JC004410.

Kaihatu, J. M., and J. T. Kirby, 1995: Nonlinear transformation of waves in finite water depth. Phys. Fluids, 7, 1903, doi:10.1063/ 1.868504 
Komen, G., L. Cavaleri, M. Donelan, K. Hasselmann, S. Hasselmann, and P. A. E. M. Janssen, 1994: Dynamics and Modelling of Ocean Waves. Cambridge University Press, 532 pp.

Long, R. B., 1973: Scattering of surface waves by an irregular bottom. J. Geophys. Res., 78, 7861-7870, doi:10.1029/ JC078i033p07861.

Lygre, A., and H. E. Krogstad, 1986: Maximum entropy estimation of the directional distribution in ocean wave spectra. J. Phys. Oceanogr., 16, 2052-2060, doi:10.1175/1520-0485(1986)016<2052: MEEOTD $>2.0 . \mathrm{CO} ; 2$.

Magne, R., K. A. Belibassakis, T. H. C. Herbers, F. Ardhuin, W. C. O'Reilly, and V. Rey, 2007: Evolution of surface gravity waves over a submarine canyon. J. Geophys. Res., 112, C01002, doi:10.1029/2005JC003035.

Mandel, L., and E. Wolf, 1995: Optical Coherence and Quantum Optics. Cambridge University Press, 1166 pp.

Miles, J. W., 1957: On the generation of surface waves by shear flows. J. Fluid Mech., 3, 185-204, doi:10.1017/S0022112057000567.

Munk, W. H., and M. A. Traylor, 1947: Refraction of ocean waves: A process linking underwater topography to beach erosion. J. Geol., 55, 1-26, doi:10.1086/625388.

O'Reilly, W. C., and R. T. Guza, 1991: Comparison of spectral refraction and refraction-diffraction wave models. J. Waterw. Port Coastal Ocean Eng., 117, 199-215, doi:10.1061/ (ASCE)0733-950X(1991)117:3(199).

— in the Southern California Bight. Coastal Eng., 19, 263-282, doi:10.1016/0378-3839(93)90032-4.

Pearman, D. W., T. H. C. Herbers, T. T. Janssen, H. D. van Ettinger, S. A. McIntyre, and P. F. Jessen, 2014: Drifter observations of the effects of shoals and tidal-currents on wave evolution in San Francisco Bight. Cont. Shelf Res., 91, 109-119, doi:10.1016/ j.csr.2014.08.011.

Pedersen, H., and O. Lokberg, 1992: Coherence and radiative energy transfer for linear surface gravity waves in water of constant depth. J. Phys., 25A, 5263-5278, doi:10.1088/0305-4470/25/ 20/009.

Phillips, O. M., 1957: On the generation of waves by turbulent wind. J. Fluid Mech., 2, 417-445, doi:10.1017/S0022112057000233.
Salmon, J. E., L. H. Holthuijsen, M. Zijlema, and G. Van Vledder, 2015: Scaling depth-induced wave-breaking in two-dimensional spectral wave models. Ocean Modell., 87, 30-47, doi:10.1016/ j.ocemod.2014.12.011.

Smit, P. B., and T. T. Janssen, 2013: The evolution of inhomogeneous wave statistics through a variable medium. J. Phys. Oceanogr., 43,1741-1758, doi:10.1175/JPO-D-13-046.1.

Stelling, G. S., and M. Zijlema, 2003: An accurate and efficient finite-difference algorithm for non-hydrostatic free-surface flow with application to wave propagation. Int. J. Numer. Methods Fluids, 43, 1-23, doi:10.1002/fld.595.

Strichartz, R., 1993: A Guide to Distribution Theory and Fourier Transforms. CRC Press, $224 \mathrm{pp}$.

Thomson, J., S. Elgar, and T. H. C. Herbers, 2005: Reflection and tunneling of ocean waves observed at a submarine canyon. Geophys. Res. Lett., 32, L10602, doi:10.1029/ 2005 GL022834.

,,--- , B. Raubenheimer, and R. T. Guza, 2007: Refraction and reflection of infragravity waves near submarine canyons. J. Geophys. Res., 112, C10009, doi:10.1029/ 2007JC004227.

Thornton, E. B., and R. T. Guza, 1983: Transformation of wave height distribution. J. Geophys. Res., 88, 5925-5938, doi:10.1029/ JC088iC10p05925.

Van der Vorst, H. A., 1992: Bi-CGSTAB: A fast and smoothly converging variant of $\mathrm{Bi}-\mathrm{CG}$ for the solution of nonsymmetric linear systems. SIAM J. Sci. Stat. Comput., 13, 631-644, doi:10.1137/0913035.

Ville, J., 1948: Theorie et applications de la notion de signal analytique. Cables Transm., 2A, 61-74.

Wigner, E., 1932: On the quantum correction for thermodynamic equilibrium. Phys. Rev., 40, 749, doi:10.1103/PhysRev.40.749.

Willebrand, J., 1975: Energy transport in a nonlinear and inhomogeneous random gravity wave field. J. Fluid Mech., 70, 113-126, doi:10.1017/S0022112075001929.

Zijlema, M., G. S. Stelling, and P. B. Smit, 2011: SWASH: An operational public domain code for simulating wave fields and rapidly varied flows in coastal waters. Coastal Eng., 58, 9921012, doi:10.1016/j.coastaleng.2011.05.015. 